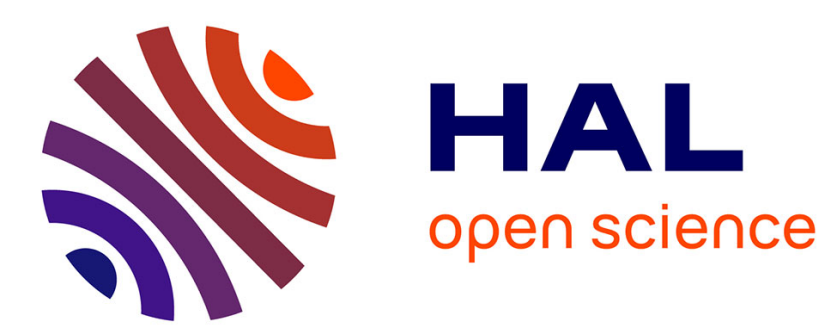

\title{
Fast, repeatable and precise magnetic actuation in ambient environments at the micrometer scale
}

\author{
Aude Bolopion, Soukeyna Bouchebout, Stéphane Regnier
}

\section{To cite this version:}

Aude Bolopion, Soukeyna Bouchebout, Stéphane Regnier. Fast, repeatable and precise magnetic actuation in ambient environments at the micrometer scale. Journal of Micro-Bio Robotics, 2017, 13 (1 - 4), pp.55 - 66. hal-02868189

\section{HAL Id: hal-02868189 \\ https://hal.science/hal-02868189}

Submitted on 15 Jun 2020

HAL is a multi-disciplinary open access archive for the deposit and dissemination of scientific research documents, whether they are published or not. The documents may come from teaching and research institutions in France or abroad, or from public or private research centers.
L'archive ouverte pluridisciplinaire HAL, est destinée au dépôt et à la diffusion de documents scientifiques de niveau recherche, publiés ou non, émanant des établissements d'enseignement et de recherche français ou étrangers, des laboratoires publics ou privés. 


\title{
Fast, repeatable and precise magnetic actuation in ambient environments at the micrometer scale
}

\author{
Aude Bolopion · Soukeyna \\ Bouchebout • Stéphane Régnier .
}

Received: date / Accepted: date

\begin{abstract}
This work aims at increasing the velocity of micrometer scale particles controlled by non contact magnetic actuation systems. The particles are placed in an ambient environment (i.e. in air) to minimize the drag forces. However this approach raises two major issues: the repeatability and the precision of position are difficult to obtain in ambient environments due to the adhesion force between the substrate and the particle. This work proposes to use first a magnetic torque to provoke in-plane rotation of the particle to overcome adhesion between the particle and the substrate. Then a magnetic force is applied to induce the movement of the particle. To ensure that the static friction is broken and that the position of the particle can be controlled precisely a current pulse actuation mode is used. A dedicated closed loop control law which controls both the amplitude and the duration of the current simultaneously is proposed to ensure accurate positioning of the particle. Speed during pulse can reach $176 \mathrm{~mm} / \mathrm{s}$ (more than 350 body lengths per second) in open loop on silicon substrates. Adhesion is overcome in $95 \%$ of the tests using the magnetic torque, compared to $66 \%$ using classical approaches. Precision of positioning of less than $20 \%$ of the size of the particle can be reached. The approach proposed in this paper is generic so that it can be easily transposed to other systems in the literature. The large number of experimental tests provides a deep understanding of the possibilities and challenges of magnetic actuation in ambient environments.
\end{abstract}

Aude Bolopion

Institut FEMTO-ST, Univ. Bourgogne Franche-Comté, CNRS, 24 rue Alain Savary, 25000

Besançon, France.

E-mail: aude.bolopion@femto-st.fr

Soukeyna Bouchebout and Stéphane Régnier

Institut des Systèmes Intelligents et de Robotique, Université Pierre et Marie Curie, CNRS UMR 7222, 4 Place Jussieu, 75005 Paris, France.

E-mail: regnier@isir.upmc.fr 
Keywords Microrobotics · non contact actuation · magnetic actuation

\section{Introduction}

Micrometer scale is the scale of biological cells, but also the scale of MEMS and NEMS components. There is thus a huge applicative interest in the manipulation of micrometer size objects. However fast, precise and repeatable positioning remains a challenge at this scale. Classical approaches, based on the use of grippers and tips, suffer from the inertia of the robotic structure which limits the manipulation speed [1] [2]. To overcome this issue remotely actuated microrobotic systems, based on remote force fields to position the microobjects, have gain a large interest these last few years [3], [4].

Among the most commonly used force fields magnetic actuation is highly attractive since important and long range forces can be produced by the coils [5], [6], [7], [8]. Rotating magnetic fields actuate helical microrobots [9] while magnetic field gradients are widely used to induce displacements of magnetic particles [8], [10]. Oscillating fields move particles with a mass-spring architecture [11] and the magnetic torque induces stick slip motion if the particles are on a substrate [12]. The motion of the particles can be controlled either in the plane (2D motion) [13], or in space [14]. The independent control of several magnetic particles using a single force field is presented in [15]. Indirect manipulation where magnetic particles are used as tools to manipulate non magnetic objects is demonstrated in [16] or in [17]. In [18], magnetic actuation controls on-board degrees of freedom, to remotely open and close grippers. However, even if some works report high velocities in specific conditions [13], [19] only very few works propose strategies to increase the velocity of the displacement of the particles on non specialized surfaces.

The goal of this article is to propose practical solutions to increase the velocity of magnetically actuated micrometer particles on non-specialized surfaces. To increase the velocity the drag forces must be minimized. To fulfill this requirement this work proposes to place the particles in ambient environments (the particles are actuated in air). However this approach raises two major issues: (i) the repeatability is difficult to obtain in ambient environments due to the adhesion between the substrate and the particle that must be overcome before a movement occurs and (ii) the precision of positioning is difficult to obtain since high amplitude magnetic force is used to reach high velocities. Most of the works dealing with magnetic actuation get rid of these issues by placing the particles in a liquid [14], [20]. Adhesion between the substrate and the particle is then highly reduced, and the viscosity of the liquid induces an important drag force that limits the velocity. This work aims at evaluating the performances that can be reached while placing the particles in air.

Two previous works proposed an original approach to increase repeatability of actuation [21], [22]. In [21] a piezoelectric actuation is used to overcome 
adhesion between the particle and the substrate. However it necessitates to apply high voltages which present the risk of electrical breakdowns. In [22] substrates of different materials are proposed and textured using clean room facilities to reduce either the contact area between the particle and the substrate or the electrostatic charges. However it requires time consuming microfabrication process of the substrate. This current work proposes a different strategy, for non specialized surfaces. First a magnetic torque is used to provoke in-plane rotation of the particle (contrary to stick-slip actuation which uses out of plane rotation) to overcome adhesion between the particle and the substrate. Then a high magnetic force is applied to induce the movement of the particle. To ensure the precision of positioning despite the application of a high magnetic force this work proposes a specific control mode, using current pulses in which high currents are applied for a short duration. Two inputs, the duration of the actuation and the current set to the coils can be controlled. In [22] the control law controls only the duration of the signal. On the contrary, in this work, a dedicated control law able to control these two inputs simultaneously is proposed and evaluated. It is based on these two proportional-integral PI controllers.

The experimental results show that a precise positioning of the particle can be reached in a limited number of steps. This works opens the door to advanced control aiming both at increasing the velocity of the control, and the complexity of the tasks performed by the micrometer size particles. Remote handling of components, or remote assembly is envisioned. From the application point of view, as it is performed in ambient environments, this actuation technique is of interest for the handling of MEMS and NEMS components as long as they are compatible with magnetic fields.

This paper is organized as follows. The experimental setup is presented in Sec. 2. Sec. 3 presents a strategy based on the magnetic torque to overcome adhesion in order to improve repeatability for magnetic actuation in ambient environments. Current pulse actuation is analyzed in Sec. 4. A dedicated closed loop control is proposed to ensure the precision of positioning. Sec. 5 concludes the paper and provides directions for future works.

\section{Magnetic actuation at the micrometer scale}

\subsection{Magnetic actuation platform}

The setup is composed of the micrometer size particle that must be controlled, and a macroscopic system dedicated to the detection and actuation modules (see Fig. 1). To generate large magnetic gradients and thus large magnetic forces a specific care has been taken to the compactness of the actuation platform. In addition, ferromagnetic cores are added to the coils.

The micrometer size particle is fabricated using clean room facilities (see [22] for details). A layer of around $25 \mu \mathrm{m}$ of nickel is made by electrodeposition 
on a sacrificial substrate. The particles are obtained by saw dicing the sample at the desired dimensions (around $500 \mu \mathrm{m} \times 300 \mu \mathrm{m} \times 25 \mu \mathrm{m}$ ). One single particle is then placed on a substrate, either on glass or on silicon. To limit dust deposited on the substrates, which is a major issue for repeatability, the particle and the substrate are placed on a sealed arena inside a controlled environment. During the assembly the temperature of the room is regulated to $24{ }^{\circ} \mathrm{C}$, and the assembly is performed in a microbiological safety cabinet (PSM airster 20, MMSI). This cabinet limits dust deposition since it is a class 100 FED STD 209E, equivalent to class 5 ISO EN 14644 cabinet.

The detection of the particle is performed by a camera. Both the position of the center of mass and the orientation of the particle are determined by classical image processing techniques (erosion, dilatation, canny filter). The actuation of the particle is ensured by four orthogonal in-plane coils that generate a magnetic field. A digital to analog National Instrument converter PCIe 6259 and a voltage-to-current amplifier are used to apply the currents determined by a PC to the coils. Two parameters can be controlled for each coil $e(e=0, . ., 3)$ : the amplitude of the current $i_{e}$ and the duration of the signal $h_{e}$. When powered the coils produce a magnetic field B. A magnetic object placed in the vicinity of the coils experiences both a magnetic force $\mathbf{F}_{m}$ and a magnetic torque $\mathbf{T}_{m}$ given by:

$$
\begin{gathered}
\mathbf{F}_{m}=\mathrm{V}(\mathbf{M} \bullet \nabla) \mathbf{B} \\
\mathbf{T}_{m}=\mathrm{VM} \times \mathbf{B}
\end{gathered}
$$

where $\mathrm{V}$ and $\mathbf{M}$ are respectively the volume and the magnetic moment of the particle. A PC running real time Xenomai/Linux controls the input of the coils and processes the images coming from the camera.

\subsection{Determination of the magnetic field}

To move the particle to a given position a magnetic force $\mathbf{F}_{m}$ must be applied. The control law computes the corresponding magnetic field $\mathbf{B}$ to be produced by the coils (Eq. (1)). Since the relation between $\mathbf{F}_{m}$ and $\mathbf{B}$ will be used for closed loop control (Sec. 4.2) the model of the magnetic field must be invertible in real time. A polynomial function approximation is used in this work. The coefficients of this function are identified from experimental measurements made by a hall sensor (GaussMeter Hirst GM08) mounted on a motorized micromanipulator. For each coil powered by a given current $\left(i_{e}=1, . ., 4 \mathrm{~A}\right)$, three measures are made at a given position $(x, y)$. Results for coil $e=0$ and $i_{0}=3 \mathrm{~A}$ are given in Fig. 2 where the dots represent the mean value of the magnetic field. For a given current, the variation of the magnetic field along the longitudinal direction of the coil $B_{x}$ can be interpolated by a 3 order polynomial function along the $x$ axis and by a 2 order polynomial function along the $y$ axis. The one representing the magnetic field along the radial direction of the coil $B_{y}$ can be interpolated by a 2 order polynomial function 


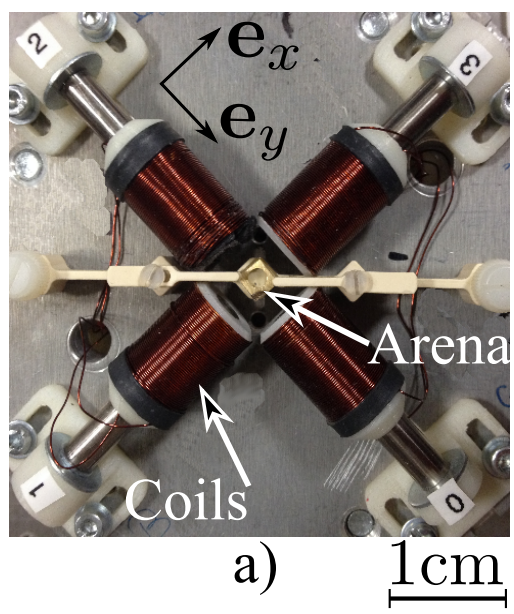

b)

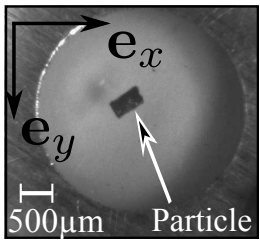

c)

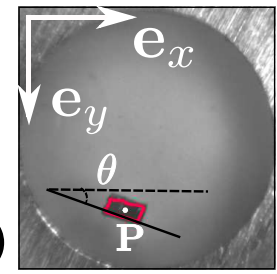

Fig. 1 Experimental platform. a) Global view of the non contact actuation platform. b) View of the arena that includes the micrometer size particle. c) Visual detection of the particle position and orientation. $\mathbf{P}$ is the position of the particle and $\theta$ is its orientation.

\begin{tabular}{ccccccc}
\hline & $1 \mathrm{~A}$ & $2 \mathrm{~A}$ & $3 \mathrm{~A}$ & $4 \mathrm{~A}$ & ${ }^{0} P_{22}[m, n]$ & $\sigma^{0} P_{22}[m, n]$ \\
\hline \hline${ }^{0} P_{22}[0,0]$ & 9,13 & 10,02 & 10,86 & 11,22 & 10,31 & 0,93 \\
${ }^{0} P_{22}[1,0]$ & $-1,32$ & $-1,15$ & $-0,95$ & $-0,80$ & $-1,06$ & 0,22 \\
${ }^{0} P_{22}[0,1]$ & $-3,34$ & $-4,00$ & $-4,61$ & $-4,92$ & $-4,22$ & 0,70 \\
${ }^{0} P_{22}[2,0]$ & 0,12 & 0,09 & 0,07 & 0,05 & 0,08 & 0,03 \\
${ }^{0} P_{22}[1,1]$ & 0,08 & 0,10 & 0,10 & 0,09 & 0,09 & 0,01 \\
${ }^{0} P_{22}[2,2]$ & 0,46 & 0,56 & 0,65 & 0,70 & 0,59 & 0,11 \\
\hline
\end{tabular}

Table 1 Values of the coefficients of polynomial ${ }^{0} P_{22}$ interpolated for different values of current. The numbers inside brackets, referring to $m$ and $n$ are the degree of the coefficient of the polynomial ${ }^{0} P_{22}$ for the $x$ and $y$ variable respectively $\left({ }^{0} \bar{P}_{22}[m, n]\right.$ refers to the coefficient of the term $\left.x^{m} y^{n}\right)$.

along both $x$ and $y$ axes. The order of the interpolation functions is chosen to fit correctly the experimental data. In addition, the magnetic field is considered proportional to the current $i_{e}$ [14]. The magnetic field produced by one coil $e$ is:

$$
B_{*}={ }^{e} P_{j k}(x, y) i_{e}
$$

where $B_{*}$ stands either for $B_{x}$ or $B_{y}, P$ is a polynomial function of order $j$, respectively $k$ along $\mathbf{e}_{x}$,respectively $\mathbf{e}_{y}$ axis. The coefficients of the polynomial functions ${ }^{e} P_{j k}$ are identified by a polynomial regression based on the least mean square technique for each value $i_{e}$. Table 1 presents the coefficients of the polynomial ${ }^{0} P_{22}$ for each value of $i_{e}$, as well as the mean value and the standard deviation. Since the values of ${ }^{0} \bar{P}_{22}[j, k]$ determined do not vary much depending on the current, the mean values are taken as the coefficients of the polynomials. Interpolated values $\hat{B}_{x}, \hat{B}_{y}$ are compared to experimental measurements in Fig. 2. The coefficients of determination are respectively 0.94 


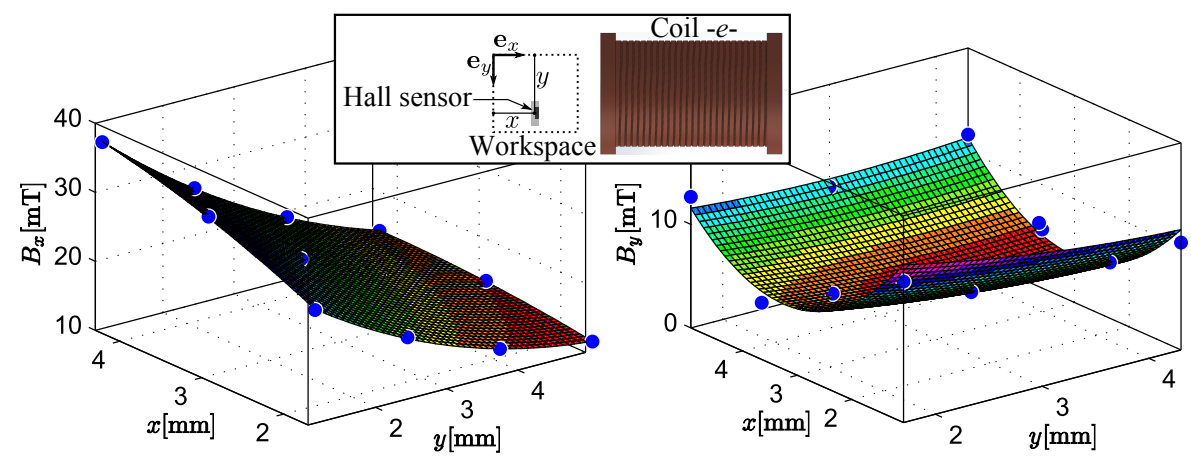

Fig. 2 Experimental (represented by the dots) and interpolated (represented by the surfaces) values $\left(B_{x}, B_{y}\right)$ for coil $e=0$ and $i_{0}=3 \mathrm{~A}$.

and 0.68 for $\hat{B}_{x}, \hat{B}_{y}$. The interpolation of $\hat{B}_{y}$ is less precise than $\hat{B}_{x}$ because the values of the radial magnetic field produced by the coil are lower than the ones of the axial magnetic field. Measurements using the hall sensor are thus less accurate. This interpolation is however considered satisfactory for closed loop control of the particle as the feedback loop will correct the variations between the values of the magnetic field predicted by the model and the real ones. If the magnetic actuation system has to be used in open loop, a more accurate model would however be required. Model-based calibration could be an interesting alternative [23].

The magnetic field produced by the three other coils can be deduced from these results by considering that the coils are orthogonal. However, to take into account the slight differences of the coils, due for example to a slightly different number of turns, the same study is performed for the three other coils.

It is supposed that the core of the coils are ideal soft-magnetic materials, that hysteresis is negligible and that the system is operated with the cores in their linear magnetization region. In that case, the magnetic field produced by the four coils is the superposition of the magnetic field produced by each coil [14]. The magnetic gradients $B_{d x}(\mathbf{P})$ and $B_{d y}(\mathbf{P})$ are obtained by the spatial derivation of this field:

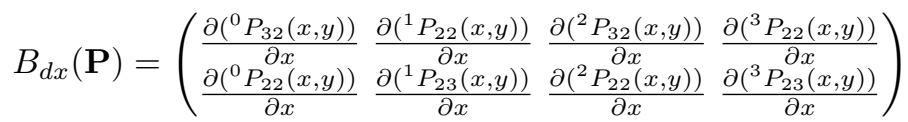

and :

$$
B_{d y}(\mathbf{P})=\left(\begin{array}{llll}
\frac{\partial\left({ }^{0} P_{32}(x, y)\right)}{\partial y} & \frac{\partial\left({ }^{1} P_{22}(x, y)\right)}{\partial y} & \frac{\partial\left({ }^{2} P_{32}(x, y)\right)}{\partial y} & \frac{\partial\left({ }^{3} P_{22}(x, y)\right)}{\partial y} \\
\frac{\partial\left({ }^{0} P_{22}(x, y)\right)}{\partial y} & \frac{\partial\left({ }^{1} P_{23}(x, y)\right)}{\partial y} & \frac{\partial\left({ }^{2} P_{22}(x, y)\right)}{\partial y} & \frac{\partial\left({ }^{3} P_{23}(x, y)\right)}{\partial y}
\end{array}\right)
$$

where $\mathbf{P}$ is the position of the particle. The magnetic force can be obtained from the previous results:

$$
\mathbf{F}_{m}=\mathbf{G}(\mathbf{P}, \theta) \mathbf{I}
$$


where $\mathbf{I}=\left[\begin{array}{llll}i_{0} & i_{1} & i_{2} & i_{3}\end{array}\right]^{T}$ is the current sent to the coils and $\mathbf{G}(\mathbf{P}, \theta)$ is defined as:

$$
\mathbf{G}(\mathbf{P}, \theta)=\left(\begin{array}{l}
V \mathbf{M}(\theta)^{T} B_{d x}(\mathbf{P}) \\
V \mathbf{M}(\theta)^{T} B_{d y}(\mathbf{P})
\end{array}\right)
$$

and $\mathbf{P}=\left[\begin{array}{ll}x & y\end{array}\right]^{T}$ is the position of the particle, $\theta$ is its orientation and $M$ is its magnetisation given by:

$$
\mathbf{M}(\theta)=\|\mathbf{M}\|[\cos (\theta) \sin (\theta)]^{T}
$$

The particle is made from electrodeposited nickel. The magnetization $\|\mathbf{M}\|$ is considered constant here and equal to $4.710^{5} \mathrm{~A} / \mathrm{m}$ according to [24].

These last equations will be used in Sec. 4.2 to compute the current I sent to the coils to produce the required magnetic force to position precisely the particle.

\section{Magnetic torque for increased repeatability}

In an ambient environment the drag forces are greatly reduced due to the low viscosity of air, which enables to generate high speed displacements. However it first necessitates to overcome the adhesion forces between the particle and the substrate, which makes repeatability a major issue. In [22] different substrates are analyzed to reduce adhesion and increase repeatability. Experiments show that the polished silicon surface presents a reduced roughness and electrical resistivity. It guarantees the highest percentage of particle displacements among the tested surfaces (glass, structured silicon and polished silicon). However, it induces a strong limitation on the substrate that must be used. To overcome this limitation, an original approach based on the application of a magnetic torque to overcome adhesion is proposed and evaluated in this section.

To demonstrate the benefit of the proposed approach based on the magnetic torque we purposely chose to perform all experimental tests of this section on a common substrate, glass, which induces issues in terms of repeatability (according to $[22]$ ).

\subsection{Proposed approach}

To break the contact between the particle and the substrate a magnetic force $\mathbf{F}_{\mathrm{m}}$ and/or a magnetic torque $\mathbf{T}_{m}$ greater than the adhesion force between the particle and the substrate $\mathbf{F}_{a d h}$ and the corresponding torque $\mathbf{T}_{a d h}$ must be applied. $\mathbf{T}_{m}$ is a function of the magnetic field whereas $\mathbf{F}_{\mathrm{m}}$ depends on the gradient of the magnetic field (Eq. (2), Eq. (1)). At a distance $d$ from the magnetic source, the amplitude of the magnetic field decreases as $d^{-3}$. The one of the gradient of the magnetic field decreases as $d^{-4}$ [25]. The torque is thus a good candidate to overcome adhesion.

The proposed approach is to decompose the actuation of a particle in two stages: 


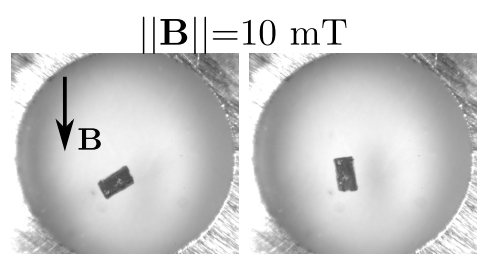

Fig. 3 Application of a magnetic torque to produce in-plane rotation of the particle to overcome adhesion. Left: initial position, right: position after the application of the magnetic field.

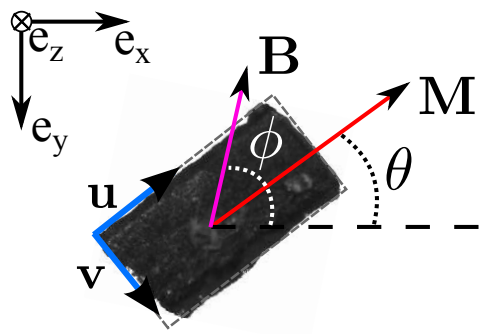

Fig. 4 Magnetisation of the particle. The magnetic moment is aligned with the long axis of the particle, in the direction $\mathbf{u}$. A magnetic field is applied with an angle $\phi$ to induce a magnetic torque on the particle along $e_{z}$.

- Stage 1: a magnetic torque is applied to break the contact between the particle and the substrate. It must thus overcome adhesion. In this stage the particle should experience an in-plane rotation around its center of mass. The center of mass should stay at the same position. Any displacements of the center of mass is referred to "undesired displacements" in the paper,

- Stage 2: a magnetic force is applied on the particle, to move it to a desired position.

The torque applied in this paper differs from stick-slip actuation since it is about the z-axis, which provokes an in-plane rotation of the particle as depicted in Fig. 3. On the contrary, stick slip motion necessitates to apply a torque about the $\mathrm{x}$ or $\mathrm{y}$-axis [12], which requires an out-of-plane coil. The setup used in this work is composed of only four in-plane coils, which prevents from performing stick slip actuation.

The torque depends on the magnetic moment $\mathbf{M}$. For soft ferromagnetic particles, this moment tends to be aligned along the longest axis of the particle (Figure 4) [26]. Based on Eq. (2) and Eq. (6) the magnetic torque can be written as:

$$
\left\|\mathbf{T}_{m}\right\|=\mathrm{V}\|\mathbf{B}\|\|\mathbf{M}\||\sin (\phi-\theta)|
$$

It depends on $\|\mathbf{B}\|$ and the $\phi-\theta$ angle. It is null for $\phi=\theta$ and increases as $\phi-\theta$ increases until it reaches $90^{\circ}$. The magnetic torque tends to align the 


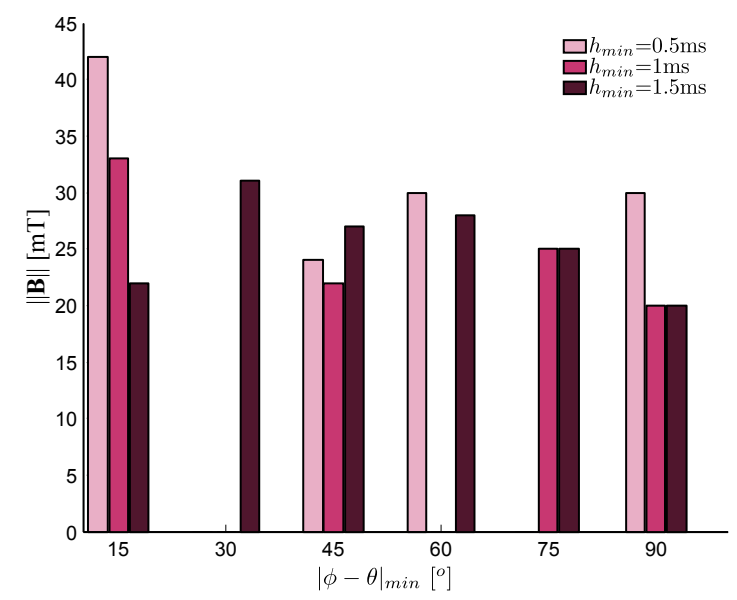

Fig. 5 Determination of the parameters $|\phi-\theta|_{\text {min }}$ and $h_{\text {min }}$. To be considered the parameters should correspond to a value of $\|\mathbf{B}\|$ lower than $35 \mathrm{mT}$. The absence of a bar (for 30, 60 and $75^{\circ}$ ) means that even after 200 trials the value of $\|\mathbf{B}\|$ still has to be increased to detach the particle.

magnetic moment along the direction of the magnetic field. It is directed along the $e_{z}$ axis.

\subsection{Tuning of the actuation parameters}

In Stage 1, to apply a torque to the particle, a magnetic field must be generated. However, as the coils are not in an Helmholtz configuration it is not possible, using the setup presented in Fig. 1, to generate a uniform magnetic field. A magnetic gradient is thus present in Stage 1. This magnetic gradient induces an undesired magnetic force, which provokes undesired displacements of the center of mass of the particle. The amplitude of the magnetic field $\|\mathbf{B}\|$, its orientation $\phi$ and the duration of the signal $h$ must be tuned to limit the undesired displacements of the particle while applying the torque able to break the contact between the particle and the substrate.

Experiments are performed for a $(\phi-\theta)$ angle comprised between $15^{\circ}$ and $90^{\circ}$. Its value varies in increments of $15^{\circ}$. Values below $0^{\circ}$ would lead to a rotation of the particle in the opposite direction, while values above $90^{\circ}$ would lead to a re-magnetisation of the particle. The duration of the signal varies from $0.5 \mathrm{~ms}$ to $1.5 \mathrm{~ms}$. Two hundred iterations are performed as follows: a magnetic field is applied with an angle $\phi$ and an amplitude $\|\mathbf{B}\|$. If the particle adheres to the substrate the amplitude $\|\mathbf{B}\|$ is increased, otherwise it is kept constant.

For each angle, Figure 5 depicts the necessary amplitude $\|\mathbf{B}\|$ to detach the particle for a given duration of application of the signal $h_{\min }$. For a given angle, the absence of a bar means that after 200 iterations the value of $\|\mathbf{B}\|$ 
still had to be increased to get a repeatable detachment of the particle. A set of parameters $\left\{\|\mathbf{B}\|, h_{m i n},|\phi-\theta|_{m i n}\right\}$ is considered as valid if the amplitude $\|\mathbf{B}\|$ converges after 150 iterations to a value lower than $35 \mathrm{mT}$. This limit is set to avoid the heating of the coils. As shown in Fig. 5 all the set of parameters for which $\|\mathbf{B}\|$ converges are acceptable except for $h_{\text {min }}=0.5 \mathrm{~ms}$, $|\phi-\theta|_{\min }=15^{\circ}$. The fact that the $0.5 \mathrm{~ms}$ pulse often fail is most probably due to the inertia of the particle. This will be verified in the next section since, according to Fig. 7, it takes around $1 \mathrm{~ms}$ before a particle starts moving while applying a force on a glass substrate, and few milliseconds when the particle is on a silicon substrate. The duration of the pulse should thus be sufficient so that the particle starts moving.

To evaluate the corresponding unwanted displacements the torque corresponding to each set of selected parameters is applied 200 times. The rotation as well as the amplitude of displacements are measured after a 1 second delay. Figure 6 gives the percentage of tests that are successful (a displacement or a rotation occurred), as well as the corresponding undesired displacements.

To limit the displacement of the center of mass in Stage 1, the duration of application of the undesired force (and thus of the magnetic torque) has to be limited. Indeed, the displacement is the double integral over time of the acceleration of the particle, which is directly linked to the force that is applied. The shorter the application of the undesired force is, the smaller the undesired displacements of the particle are. However, long duration of application of the torque corresponds also to the highest percentage of successful tests. In this work, it is chosen to place emphasis on the criterion of successful tests as undesired displacements can be corrected by closed loop control. The selected parameters are thus an angle of $90^{\circ}$ and a duration of the signal $h_{\min }=$ $1.5 \mathrm{~ms}$. The corresponding $\|\mathbf{B}\|$ is equal to $20 \mathrm{mT}$ according to Fig. 5. They correspond to the highest success rate: $87 \%$ of successful tests.

\subsection{Validation of the approach to increase repeatability}

This section compares two approaches to induce a movement to the particle:

- the classical approach which consists in applying a force $\mathbf{F}_{m}$ to induce the displacement,

- the proposed approach which consists in first applying a magnetic torque to break the adhesion between the particle and the substrate, and then a magnetic force $\mathbf{F}_{m}$ to induce the displacement.

For each approach the magnetic force $\mathbf{F}_{m}$ is the same, and is proportional to $\|\nabla \mathbf{B}\|=4 \mathrm{mT} / \mathrm{mm}$. The magnetic torque is applied with the parameters selected in the previous section $\left(|\phi-\theta|_{\min }=90^{\circ}, h_{\min }=1.5 \mathrm{~ms},\|\mathbf{B}\|=20 \mathrm{mT}\right)$.

Each approach is tested 100 times. The initial and final positions are set randomly. The displacement of the particle is measured after a 1 second delay. It might happen that some configurations are such that the classical and the 


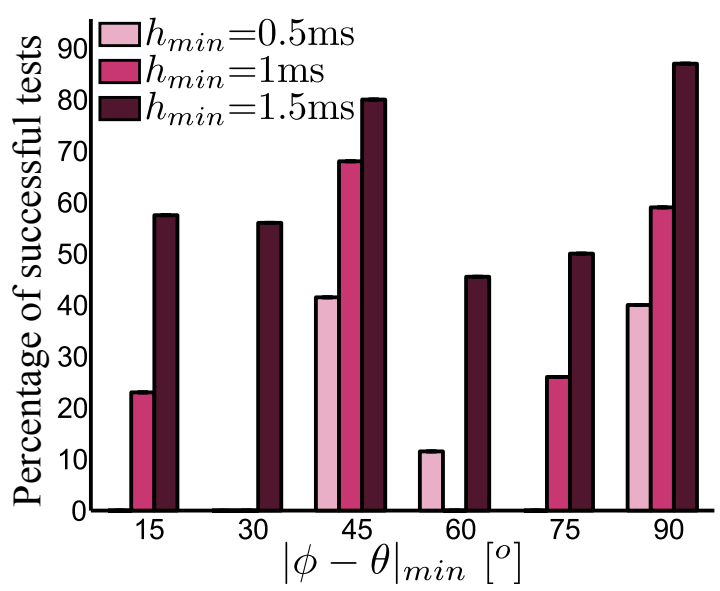

(a) Percentage of successful tests (a movement occurs, which proves that adhesion has been overcome).

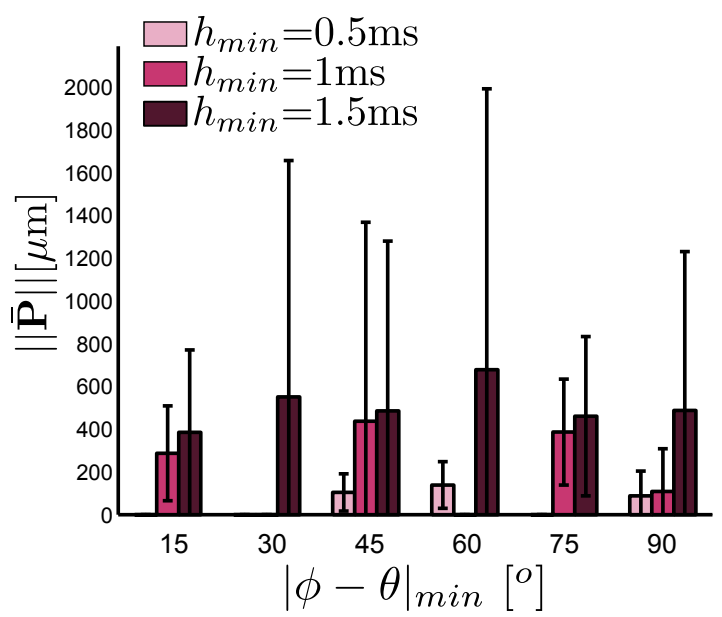

(b) Average values of the amplitude of the displacements for each values $\left(|| \mathbf{B} \|,|\phi-\theta|_{\min }, h_{\min }\right)$

Fig. 6 Evaluation of the torque actuation for the values $|\phi-\theta|_{\text {min }}$ and $h_{\text {min }}$ selected. 200 trials are performed for each set of parameters. (a) represents for each set of parameters the percentage of tests, among the 200 trials, for which a displacement or a rotation of the particle occurred. (b) represents the mean value and the standard deviation of the undesired displacements of the center of mass of the particle.

proposed approach are similar, in case the displacement and the orientation of the particle are 90 degrees apart. In that case a torque is also applied to the particle in the classical approach. However, the high number of experiments enable to get statically representative results.

Results are given in Table 2. A displacement occurs in $95 \%$ of the tests while using the magnetic torque in addition to the force, compared to $66 \%$ using the classical approach. The proposed approach is indeed suitable to 
increase the repeatability of the actuation in ambient environments. However, as expected, it provokes larger displacements (mean value $\|\overline{\mathbf{P}}\|$ ), with a larger standard deviation $\left(\sigma_{\mathbf{P}}\right)$. A closed loop control must be implemented to correct these unwanted displacements.

\begin{tabular}{c||c||cc}
\hline Actuation technique & \% of success & \multicolumn{3}{c}{ Unwanted displacements } \\
& & $\|\overline{\mathbf{P}}\|[\mu \mathrm{m}]$ & $\sigma_{\|\mathbf{P}\|}[\mu \mathrm{m}]$ \\
\hline \hline Classical approach & 66 & 158 & 239 \\
Proposed approach & 95 & 613 & 348 \\
\hline
\end{tabular}

Table 2 Comparison between the classical approach for which only a magnetic force is applied and the proposed approach that uses both a magnetic torque to overcome adhesion and a magnetic force to induce the displacement of the particle.

\section{Current pulse actuation}

To ensure a break of static friction a magnetic torque is applied to the particle, as well as a high amplitude force. However, to ensure the precision of positioning this high amplitude force must be applied for a short duration. A specific actuation mode is proposed in this paper based on current pulses to limit the time during which the coils are powered. The relevance of this approach is first analyzed experimentally in open loop. To ensure the precision of positioning a closed loop control law dedicated to the specificity of current pulse actuation is presented.

\subsection{Open loop current pulse actuation}

The proposed actuation mode by current pulses is evaluated experimentally. To get a deep understanding of the trajectory of the particle a specific high speed camera, Fastcam SA1.1 from Photron [27] is used in this paragraph. It records $5000 \mathrm{fps}$, but only off-line processing is possible. Figure 7 presents the results. On a glass substrate the mean velocity during one pulse corresponds to $55 \mathrm{~mm} / \mathrm{s}$. Significantly higher velocities can be obtained on a silicon substrate, since average speed during pulse can reach $176 \mathrm{~mm} / \mathrm{s}$, more than 350 body lengths per second (which means that the displacement of the particle in 1s corresponds to 350 times its size). These velocities can be compared to ones of classical magnetic actuation systems at micrometer scale [13], [19]. Velocities up to $20 \mathrm{~mm} / \mathrm{s}$ (40 body lengths per second) are reached in an ambient environment in [11]. In [28], $74 \mathrm{~mm} / \mathrm{s}$ (128 body lengths per second) are obtained. Velocities inside liquids are generally smaller due to drag forces. However high velocities can be obtained in specific conditions, such as in [19] which reports $556 \mathrm{~mm} / \mathrm{s}$ (1390 body lengths per second). The tests performed in this paper use low amplitude currents $(i=3 \mathrm{~A})$ as their goal is only to demonstrate the 


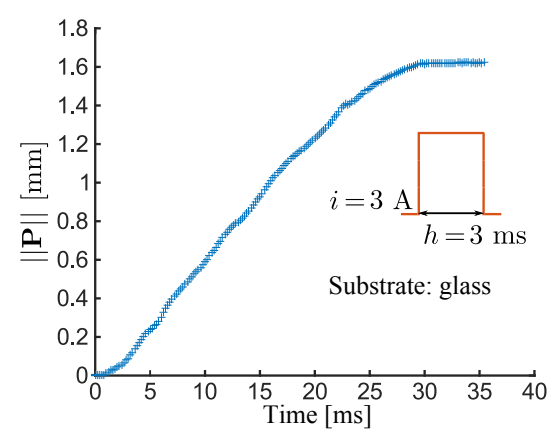

(a) Glass substrate

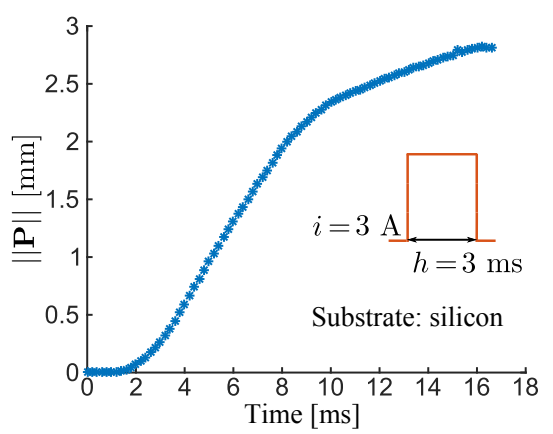

(b) Silicon substrate

Fig. 7 Position of the particle as a function of time when a single coil is powered $(i=3 \mathrm{~A}$, $h=3 \mathrm{~ms}$ ). A high frequency camera (5000 fps) records its trajectory for different substrates. Speed during pulse up to $176 \mathrm{~mm} / \mathrm{s}$ are reached.

relevance of the approach. However velocities higher than what is commonly found in the literature in ambient environments are reached.

In addition it can be noticed that even if the duration of the current pulse is only a few milliseconds $(h=3 \mathrm{~ms})$ the particle movement lasts several tens of milliseconds due to inertia. In ambient environments, a short but high amplitude force must be applied to overcome adhesion. As long as the particle is detached from the substrate, the quasi absence of drag forces enables to generate large displacements, even after the magnetic force has been switched off. This consideration highlights the relevance of current pulse actuation in ambient environments.

\subsection{Closed loop control for current pulse actuation}

To ensure the precision of the position of the particle closed loop control is implemented. In this paragraph a silicon substrate is used as the velocity is higher than on a glass substrate. For this first work it is considered that repeatability on a polished silicon substrate is sufficient, as mentioned in [22], so the classical approach, which consists in applying only a magnetic force, is implemented.

The current pulse actuation mode necessitates a dedicated controller able to control simultaneously two inputs, the amplitude of the current set to the coils and the duration of the signal. The proposed control scheme is depicted in Figure 8. Two independent proportional integral controllers (gain $K_{p}$ and $K_{i}$ ) controls respectively $i_{e}$ and $h_{e}$ for each coil. The computed current pulses are set to the coils. They generate a magnetic force during the computed duration $h$. This induces the movement of the particle. The new position $P$ of the particle is used as a feedback. The two independent controllers, which control 


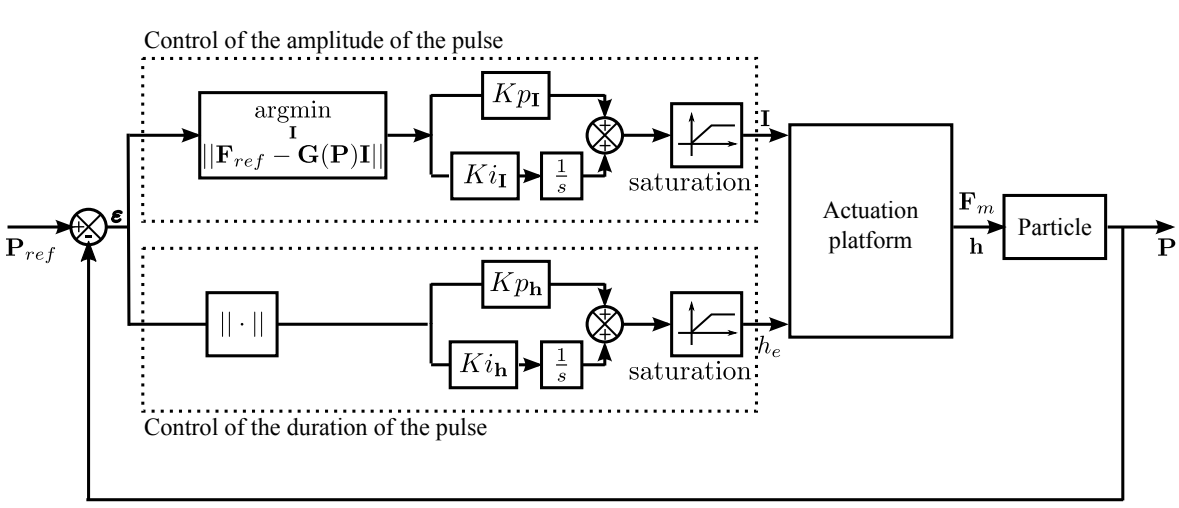

Fig. 8 Block diagram of the closed loop control of the position of the particle

respectively the amplitude of the pulse and its duration, are detailed below.

\subsubsection{Controller dedicated to control the amplitude of the pulse}

This first controller must compute the current to be set to the coils based on the error of position of the particle.

The direct relation between the current set to the coils and the magnetic force produced can be derived from Eq. (4)which provides the magnetic force as a function of the magnetic gradient. The magnetic gradient can be obtained from the magnetic field identified in Eq. (3). However this relation is non linear. Indeed, for a given current $i_{e}$, the force $\mathbf{F}_{m}$ that will be applied to the particle depends on its position relative to the actuated coil. To use a PI controller a different input should be used. One intuitive choice is to use the desired force $\mathbf{F}_{\text {ref }}$ that should be applied to the particle.

This force $\mathbf{F}_{\text {ref }}$ must be computed from the error of positioning $\epsilon$. However, it is a real issue to know the exact force $\mathbf{F}_{\text {ref }}$ that should be applied to perform a given displacement. This is especially true in this article since the experiments are performed in ambient environments, which means that the particle is actuated in air and the environmental conditions are not controlled. Adhesion between the substrate and the particle is unknown and varies greatly depending on these experimental conditions (humidity, cleanliness of the substrate ...). The estimation of this force is still an active research area, and it is thus out of the scope of that article [4]. In this work, since the position of the particle is controlled in closed-loop, it is not necessary to know the exact value of the force. It is considered that $\mathbf{F}_{r e f}$ is proportional to the error $\epsilon$ between the desired and the actual position of the particle (the proportional gain is comprised in the $K_{p I}$ and $K_{i I}$ gains of the controller). The feedback loop will correct the errors induced by the lack of a precise model. 


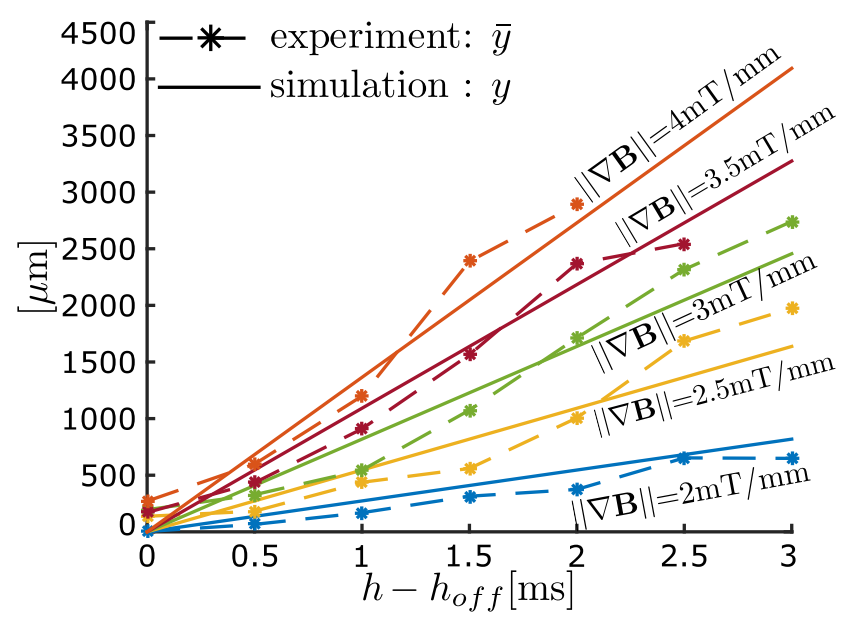

Fig. 9 Displacement of the particle as a function of the duration of the input signal for different values of the magnetic field.

The currents $i_{e}$ will then be computed from the desired force $\mathbf{F}_{\text {ref }}$ (Eq.(3) and (4)):

$$
\left\{\begin{array}{c}
\mathbf{F}_{\text {ref }}-\mathbf{G}(\mathbf{P}, \theta) \mathbf{I}=\mathbf{0}_{2} \\
i_{e}>0 \text { and } i_{e}<i_{\max } \forall e=0,1,2,3
\end{array}\right.
$$

For technical reasons the following constraints will be taken into account: the current is limited to $i_{\max }=6 \mathrm{~A}$ (corresponding to the saturation block in Fig. 8 ), and no more than two coils will be powered simultaneously. The solution of the equation is approximated using the standard least squares method to compute the current $\mathbf{I}_{M C}$ :

$$
\mathbf{I}_{M C}=\underset{\mathbf{I}}{\operatorname{argmin}}\left\|\mathbf{F}_{r e f}-\mathbf{G}(\mathbf{P}, \theta) \mathbf{I}\right\|
$$

Based on this equation the controller can compute the current that should be set to the coils to perform the desired displacement.

\subsubsection{Controller dedicated to control the duration of the pulse}

The second controller defines the duration of the signal. The displacement of a particle that undergoes a magnetic force during a time interval $h$ is identified experimentally. Fig. 9 presents the data obtained from 10 trials. For duration below $h_{\text {off }}=0.5 \mathrm{~ms}$ there is no displacement (corresponding to the saturation block in Fig. 8). It can be seen that the relation between the displacement and the duration of the signal $\left(h-h_{o f f}\right)$ is linear. This input can be used in combination with a PI controller.

This second controller can thus compute the duration of the pulse necessary to perform the desired displacement. 


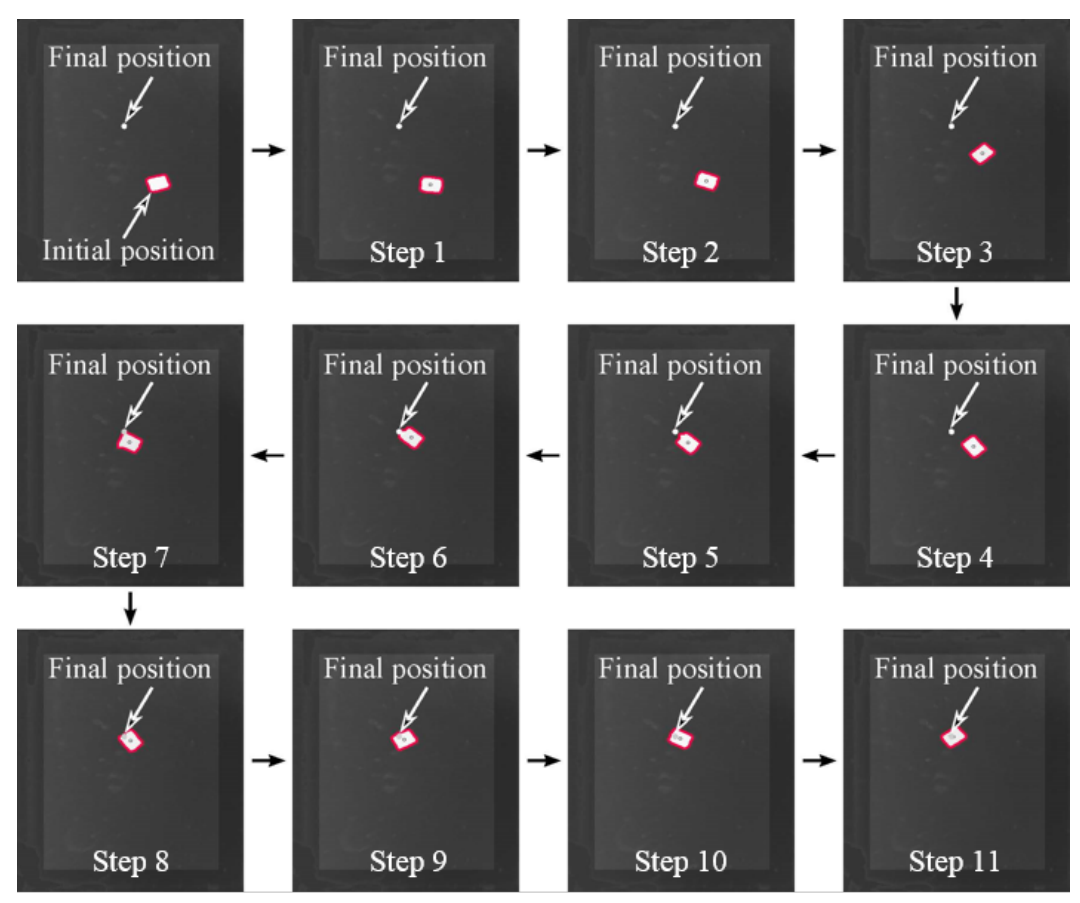

Fig. 10 Closed loop control of the position of the particle. Each image corresponds to one actuation step. The particle size is $470 \mu \mathrm{m} \times 300 \mu \mathrm{m} \times 25 \mu \mathrm{m}$. Video is available as supplementary material.

\subsubsection{Experimental validation of the control law}

Experiments are performed to validate the control law. Ten tests are made during which the initial and final positions of the particle are set randomly. The experiments are stopped as soon as the error between the desired and the actual position are less than $20 \%$ of the size of the particle. The experiments are performed on a silicon substrate. Since the repeatability on a polished silicon substrate is sufficient, as mentioned in [22], the classical approach, which consists in applying only a magnetic force, is implemented.

An example of a positioning test is given in Fig. 10 where the position of the particle for each step is recorded. Fig. 11 represents the corresponding errors along the $x$ and $y$ axes for a given step. The mean value of the final positioning error is $87 \mu \mathrm{m}$, which represents less than $20 \%$ of the size of the particle. Around 15 actuation steps are necessary. These experiments show that precise positioning can be reached in a limited number of steps using the proposed control scheme. 

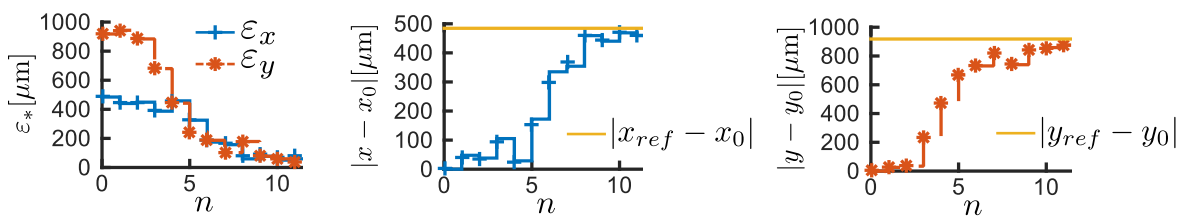

Fig. 11 Position of the particle along the $x$ and $y$ axes and related errors during the experiment depicted in Fig. 10.

\subsection{Discussion}

The goal in this paper is to propose practical solutions to perform fast, repeatable and precise magnetic actuation of micrometer size particles. The experimental results show that applying a torque to break the contact between the substrate and the particle enables to increase repeatability, and that the control scheme dedicated to pulse actuation enables to perform precise positioning. These first results are highly promising. To go further several points can be improved. In particular the system could be modeled more accurately, and the control law could be modified.

Concerning the model, in this paper the magnetization of the particle is considered constant (Sec. 2.2).However, since the value of the magnetization depends on the properties of the object and its fabrication process, it might be interesting to measure directly the magnetization of each particle using a VSM (Vibrating Sample Magnetometer). Alternatively this value can be identified based on open loop position response of a particle actuated by coils [13]. Using one of these technique would improve the accuracy of the dynamic model of the object, and could be used to improve the performances of the actuation system. In this paper, the particle is controlled in closed loop. This is why an approximated value of magnetization is sufficient since the feedback loop will correct for the inaccuracy in the estimation of the magnetization.

Concerning the control, the closed loop control presented in Section 4.2has been demonstrated on a silicon substrate because the velocity is higher than on a glass substrate. For this first work it is considered that repeatability on a polished silicon substrate is sufficient, as mentioned in [22].The classical approach, which consists in applying only a magnetic force, is implemented. The control law should be slightly modified as follows to include the strategy based on torque actuation to break the contact between the substrate and the particle in case adhesion prevents from performing the experiments. The torque has to be applied each time adhesion between the substrate and the particle prevents the particle from moving when a force is applied on it. Most of the time it is sufficient to apply it only once, at the beginning of the experiment. Adhesion between the particle and the substrate depends on the duration of the contact. However, this dependency is still an open research area [4].To ensure an efficient actuation despite this lack of knowledge about adhesion one 
solution is to monitor at each step if a displacement occurred or not after the application of the force. If the particle did not move a torque should be applied. This implies to slightly modify the block diagram of Fig. 8. On the feedback loop a comparison should be made between the position of the particle at step $k$ and the one at step $k-1$. If the position is different, nothing special is done. On the contrary, if the position is the same two cases must be considered. Either the applied force $F_{m}$ at step $k$ was null (the particle is not supposed to move), then nothing is done. Else, if the force applied at step $k$ is not null but the position at step $k$ is the same as the one at step $k-1$ this means that adhesion prevented the particle from moving. A torque should be applied to the particle to break the contact between the particle and the substrate, as explained in Sec. 3 .

The torque is applied to the direction $|\phi-\theta|_{\min }=90^{\circ}$,no matter what the direction of the desired displacement is. Then the force $F_{m}$ should be applied to the particle to move it to the desired position. The magnetic force depends on the gradient of the magnetic field. However, depending on the relative position of the particle, the desired position and the orientation of the particle, the particle might also experience a magnetic torque at this stage. For example, if the goal position is in the same direction as the magnetization of the particle, the magnetic torque will be applied to the direction $|\phi-\theta|_{\min }=90^{\circ}$ (first stage). In the second stage, a magnetic force will be applied. However, since the orientation of the particle has been modified, the desired position is orthogonal to the magnetization of the particle. The magnetic force will thus also induce a torque to re-orient the particle. In this work orientation of the particle is not controlled. This issue could be considered in future works by using a low amplitude magnetic field set in the direction of the desired orientation. The low amplitude will minimize the displacements of the particle while enabling its rotation when the final position is reached.

At the moment the control is performed sequentially: an actuation signal is sent to the platform and the detection of the particle is performed only after 100 milliseconds. This strategy has been adopted since the inertia of the particle induces a displacement of the particle long after the actuation signal has been stopped (see Fig. 7). In the future predictive control could be investigated to perform trajectory tracking. The goal of the generalized predictive control is to find the optimal future control actions that drive the future process output to track the reference trajectory as closely as possible based on the system model in the presence of system constraints and disturbances [29]. In addition, a linear closed loop control has been implemented. Despite its simplicity the proportional-integral-derivative controller remains widely used in the literature to control magnetically actuated micrometer size particles [30], [31], [32], [33], [13]. In this paper, the experiments show that precise positioning can be reached in a limited number of steps using the proposed proportional-integral control scheme. However advanced control laws would undoubtedly improve the control. Since the magnetic field is non linear, control laws dedicated to non linear systems should be considered in future works. In particular gain 
scheduling could be investigated. Gain scheduling is an approach to control non-linear systems that uses a family of linear controllers, each of which provides satisfactory control for a different operating point of the system [34].The results of this first work on PI controllers would serve as a basis to implement this control law.

\section{Conclusion and future works}

This work aims at proposing practical solutions to increase the velocity of magnetically actuated particles on non-specialized surfaces. The particles are manipulated in an ambient environment (i.e. they are placed on a substrate in air), to limit the viscous coefficient. However adhesion is a major issue. A strategy based on the use of the magnetic torque and a high amplitude magnetic force to overcome adhesion forces on non specialized substrates has been proposed to increase repeatability. Adhesion is overcome in $95 \%$ of the tests when a magnetic torque is applied, compared to $66 \%$ without the torque. To generate a high magnetic force while ensuring the precision of positioning high currents must be set to the coils during a short duration. The closed loop control scheme proposed takes into account this specificity as both the duration of the pulse and its amplitude are controlled simultaneously. After linearisation of the system two PI controllers are implemented. The particle is positioned with an error of less than $90 \mu \mathrm{m}$ (20\% of its size), and less than 16 actuation steps are necessary. Experiments performed on a single current pulse have confirmed that high velocities can be obtained in such environments, reaching speed during pulse in the order of $176 \mathrm{~mm} / \mathrm{s}$ (more than 350 body lengths per second). These results highlight the great potential of the system since these velocities are higher than what is commonly found in the literature.

This work provides key elements to get high speed actuation systems. The proposed solutions are all independent from the used setup, by opposition to classical approaches that necessitate specialised surfaces. This work on high speed control of micrometer size components in ambient environments will pave the way for future handling techniques of MEMS and NEMS based on non contact actuation. Future works include the development of strategies to manipulate non magnetic components with the magnetic particle. An actuated tool, such as a thermal microgripper, will be added to the particle to perform microassembly tasks.

Acknowledgements This work has been supported by the Labex ACTION project (contract "ANR-11-LABX-01-01"), by the "Région Franche Comté" and by the French RENATECH network and its FEMTO-ST technological facility.

\section{References}

1. M. Gauthier and S. Régnier, Robotic micro-assembly. John Wiley \& Sons, 2011. 
2. M. Kharboutly and M. Gauthier, "High speed closed loop control of a dielectrophoresisbased system," in IEEE International Conference on Robotics and Automation, 2013, pp. 1446-1451.

3. S. Chowdhury, W. Jing, and D. J. Cappelleri, "Controlling multiple microrobots: recent progress and future challenges," Journal of Micro-Bio Robotics, vol. 10, no. 1-4, pp. $1-11,2015$.

4. N. Chaillet and S. Régnier, Microrobotics for micromanipulation. John Wiley \& Sons, 2013.

5. S. Bouchebout, A. Bolopion, J.-O. Abrahamians, and S. Régnier, "An overview of multiple dof magnetic actuated micro-robots," Journal of Micro-Nano Mechatronics, vol. 7 , no. 4, pp. 97-113, 2012.

6. E. Diller and M. Sitti, "Untethered magnetic micromanipulation," Micro-and Nanomanipulation Tools, 2015.

7. L. Feng, B. Turan, U. Ningga, and F. Arai, "Three dimensional rotation of bovine oocyte by using magnetically driven on-chip robot," in IEEE/RSJ International Conference on Intelligent Robots and Systems, 2014, pp. 4668-4673.

8. I. S. Khalil, P. Ferreira, R. Eleutério, C. L. de Korte, and S. Misra, "Magnetic-based closed-loop control of paramagnetic microparticles using ultrasound feedback," in IEEE International Conference on Robotics and Automation, 2014, pp. 3807-3812.

9. A. W. Mahoney, N. D. Nelson, K. E. Peyer, B. J. Nelson, and J. J. Abbott, "Behavior of rotating magnetic microrobots above the step-out frequency with application to control of multi-microrobot systems," Applied Physics Letters, vol. 104, no. 14, p. 144101, 2014.

10. N. A. Torres and D. O. Popa, "Cooperative control of multiple untethered magnetic microrobots using a single magnetic field source," in IEEE International Conference on Automation Science and Engineering, 2015, pp. 1608-1613.

11. H.-W. Tung, M. Maffioli, D. R. Frutiger, K. M. Sivaraman, S. Pane, and B. J. Nelson, "Polymer-Based Wireless Resonant Magnetic Microrobots," IEEE transactions on Robotics, vol. 30, no. 1, pp. 26-32, 2014.

12. S. Floyd, C. Pawashe, and M. Sitti, "An untethered magnetically actuated micro-robot capable of motion on arbitrary surfaces," in IEEE International Conference on Robotics and Automation, 2008, pp. 419-424.

13. M. Dkhil, M. Kharboutly, A. Bolopion, S. Régnier, and M. Gauthier, "Closed-loop control of a magnetic particle at the air - liquid interface," IEEE Transactions on Automation Science and Engineering, vol. 14, no. 3, pp. 1387-1399, 2017.

14. M. P. Kummer, J. J. Abbott, B. E. Kratochvil, R. Borer, A. Sengul, and B. J. Nelson, "OctoMag: An Electromagnetic System for 5-DOF Wireless Micromanipulation," IEEE Transactions on Robotics, vol. 26, no. 6, pp. 1006-1017, Dec. 2010.

15. E. Diller, J. Giltinan, P. Jena, and M. Sitti, "Three dimensional independent control of multiple magnetic microrobots," in IEEE International Conference on Robotics and Automation, 2013, pp. 2576-2581.

16. Z. Ye, E. Diller, and M. Sitti, "Micro-manipulation using rotational fluid flows induced by remote magnetic micro-manipulators," Journal of Applied Physics, vol. 112, no. 6 , p. $064912,2012$.

17. A. G. El-Gazzar, L. E. Al-Khouly, A. Klingner, S. Misra, and I. S. Khalil, "Non-contact manipulation of microbeads via pushing and pulling using magnetically controlled clusters of paramagnetic microparticles," in IEEE/RSJ International Conference on Intelligent Robots and Systems, 2015, pp. 778-783.

18. S. E. Chung, X. Dong, and M. Sitti, "Three-dimensional heterogeneous assembly of coded microgels using an untethered mobile microgripper," Lab on a Chip, vol. 15, no. 7, pp. 1667-1676, 2015.

19. H. Salmon, L. Couraud, and G. Hwang, "Swimming property characterizations of magnetic polarizable microrobots," IEEE International Conference on Robotics and Automation, pp. 5520-5526, 2013.

20. M. Sitti, H. Ceylan, W. Hu, J. Giltinan, M. Turan, S. Yim, and E. Diller, "Biomedical applications of untethered mobile milli/microrobots," Proceedings of the IEEE, vol. 103, no. 2, pp. 205-224, 2015.

21. I. Ivan, G. Hwang, J. Agnus, N. Chaillet, and S. Régnier, "Nist and ieee challenge for magpier: The fastest mobile microrobots in the world," IEEE Robotics Automation Magazine, vol. 20, no. 2, pp. 63-70, 2013. 
22. S. Bouchebout, A. Bolopion, M. Gauthier, and S. Régnier, "Position control of a ferromagnetic micro-particle in a dry environment," in IEEE/ASME International Conference on Advanced Intelligent Mechatronics, 2014, pp. 1-6.

23. A. J. Petruska, J. Edelmann, and B. J. Nelson, "Model-based calibration for magnetic manipulation," IEEE Transactions on Magnetics, vol. 53, no. 7, pp. 1-6, 2017.

24. K. Mulyokov, G. Korznikov, R. Abdulov, and R. Valiev, "Magnetic hysteretic properties of submicron grained nickel and their variations upon annealing," Journal of magnetism and magnetic materials, vol. 89, no. 1-2, pp. 207-213, 1990.

25. J. J. Abbott, K. E. Peyer, M. C. Lagomarsino, L. Zhang, L. X. Dong, I. K. Kaliakatsos, and B. J. Nelson, "How should microrobots swim?." The International Journal of Robotics Research, vol. 28, no. 11-12, pp. 1434-1447, 2009.

26. J. J. Abbott, Z. Nagy, F. Beyeler, and B. J. Nelson, "Robotics in the small, part i: Microrobotics," IEEE Robotics and Automation Magazine, vol. 14, no. 2, pp. 92-103, 2007.

27. "High speed camera products - photron, www.photron.com," 2015.

28. W. Jing, X. Chen, S. Lyttle, Z. Fu, Y. Shi, and D. J. Cappelleri, "A magnetic thin film microrobot with two operating modes," in IEEE International Conference on Robotics and Automation, 2011, pp. 96-101.

29. D. Clarke, C. Mohtadi, and P. Tuffs, "Generalized predictive control part i. the basic algorithm," Automatica, vol. 23, no. 2, pp. 137 - 148, 1987.

30. D. R. Frutiger, K. Vollmers, B. E. Kratochvil, and B. J. Nelson, "Small, fast, and under control: wireless resonant magnetic micro-agents," The International Journal of Robotics Research, vol. 29, no. 5, pp. 613-636, 2010.

31. E. B. Steager, M. Selman Sakar, C. Magee, M. Kennedy, A. Cowley, and V. Kumar, "Automated biomanipulation of single cells using magnetic microrobots," The International Journal of Robotics Research, vol. 32, no. 3, pp. 346-359, 2013.

32. S. Schürle, M. S. Sakar, A. Meo, J. Möller, B. E. Kratochvil, C. S. Chen, V. Vogel, and B. J. Nelson, "Three-dimensional, automated magnetic biomanipulation with subcellular resolution," in IEEE International Conference on Robotics and Automation, 2013, pp. $1452-1457$.

33. J. Giltinan, E. Diller, and M. Sitti, "Programmable assembly of heterogeneous microparts by an untethered mobile capillary microgripper," vol. 16, no. 22, pp. 4445-4457, 2016.

34. J. S. Shamma, Gain Scheduling. John Wiley \& Sons, Inc., 2001. 